\title{
NuSTAR reveals the extreme properties of the super-Eddington accreting supermassive black hole in PG 1247+267
}

G. Lanzuisi $i^{1,2}$, M. Perna ${ }^{1,2}$, A. Comastri ${ }^{2}$, M. Cappi $^{3}$, M. Dadina ${ }^{3}$, A. Marinucci ${ }^{4}$, A. Masini ${ }^{1,2}$, G. Matt $^{4}$, F. Vagnetti ${ }^{5}$, C. Vignali ${ }^{1,2}$, D. R. Ballantyne ${ }^{6}$, F. E. Bauer ${ }^{7,8,9}$, S. E. Boggs ${ }^{10}$, W. N. Brandt ${ }^{11,12,13}$, M. Brusa ${ }^{1,2}$, F. E. Christensen ${ }^{14}$, W. W. Craig ${ }^{10,15}$, A. C. Fabian ${ }^{16}$, D. Farrah ${ }^{17}$, C. J. Hailey ${ }^{18}$, F. A. Harrison ${ }^{19}$, B. Luo ${ }^{20,21}$, E. Piconcelli ${ }^{22}$, S. Puccetti ${ }^{23,22}$, C. Ricci ${ }^{7}$, C. Saez ${ }^{24}$, D. Stern ${ }^{25}$, D. J. Walton ${ }^{19,25}$, and W. W. Zhang ${ }^{26}$

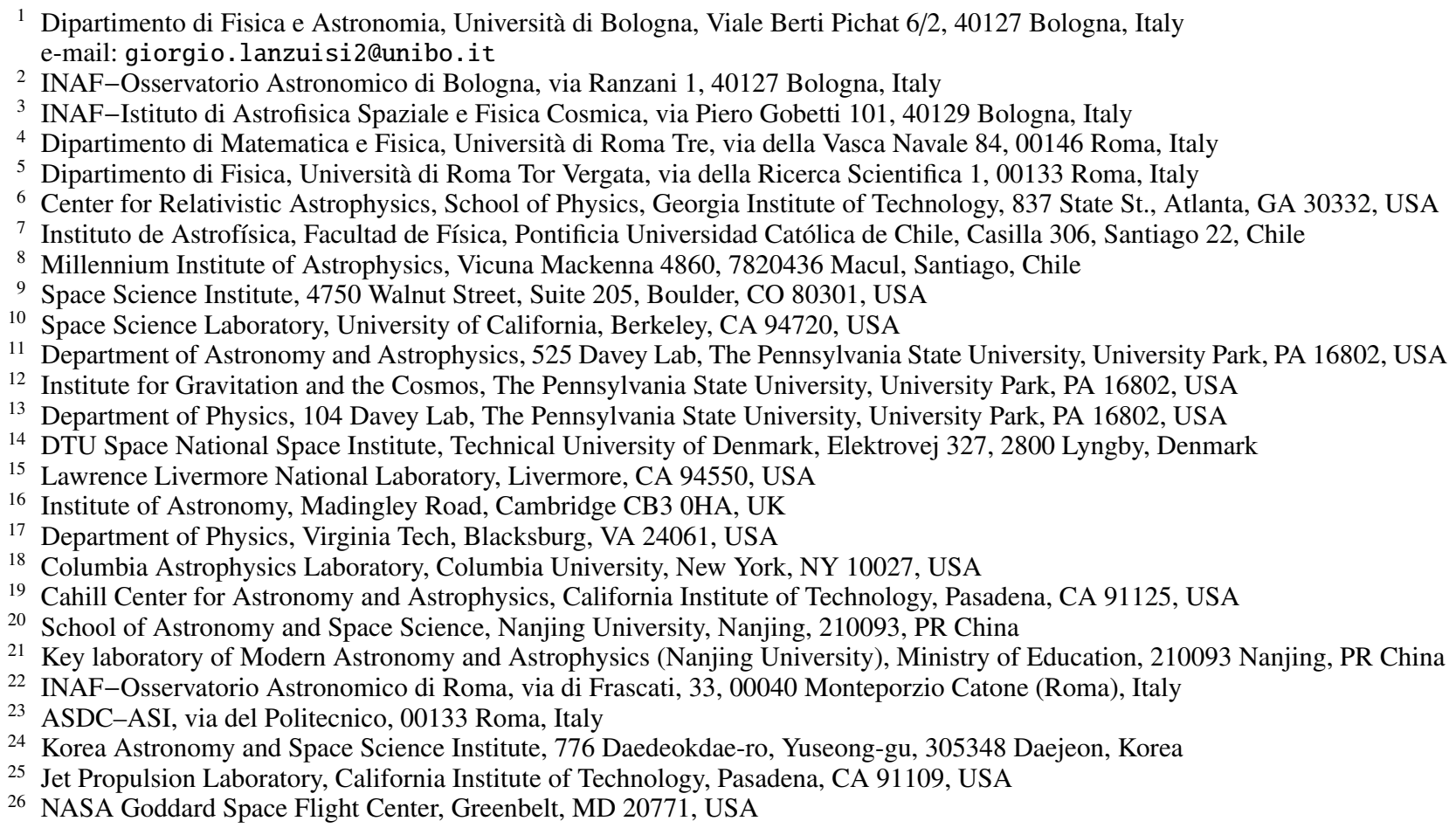

\section{ABSTRACT}

PG1247+267 is one of the most luminous known quasars at $z \sim 2$ and is a strongly super-Eddington accreting supermassive black hole (SMBH) candidate. We obtained NUSTAR data of this intriguing source in December 2014 with the aim of studying its high-energy emission, leveraging the broad band covered by the new NUSTAR and the archival XMM-Newton data. Several measurements are in agreement with the super-Eddington scenario for PG1247+267: the soft power law $(\Gamma=2.3 \pm 0.1)$; the weak ionized Fe emission line; and a hint of the presence of outflowing ionized gas surrounding the SMBH. The presence of an extreme reflection component is instead at odds with the high accretion rate proposed for this quasar. This can be explained with three different scenarios; all of them are in good agreement with the existing data, but imply very different conclusions: i) a variable primary power law observed in a low state, superimposed on a reflection component echoing a past, higher flux state; ii) a power law continuum obscured by an ionized, Compton thick, partial covering absorber; and iii) a relativistic disk reflector in a lamp-post geometry, with low coronal height and high BH spin. The first model is able to explain the high reflection component in terms of variability. The second does not require any reflection to reproduce the hard emission, while a rather low high-energy cutoff of $\sim 100 \mathrm{keV}$ is detected for the first time in such a high redshift source. The third model require a face-on geometry, which may affect the SMBH mass and Eddington ratio measurements. Deeper X-ray broad-band data are required in order to distinguish between these possibilities.

Key words. galaxies: active - galaxies: nuclei - quasars: individual: PG 1247+267 - accretion, accretion disks 


\section{Introduction}

The Eddington luminosity (expressed as $L_{\mathrm{Edd}}=4 \pi G M m_{\mathrm{p}} c / \sigma_{\mathrm{T}}$ ) is an approximate upper bound to the total luminosity $L_{\mathrm{bol}}$ that can be radiated by a compact object of mass $M$, set by the equilibrium between the radiation pressure acting outward and the gravitational force acting inward, for a spherically symmetric geometry (Eddington 1916). The limit of $L_{\mathrm{bol}} / L_{\mathrm{Edd}}$ (hereafter $\left.\lambda_{\text {Edd }}\right)=1$ is thought to regulate the growth of supermassive black holes (SMBHs) over cosmic time and is observed to hold both at low (e.g., Schulze \& Wisotzki 2010) and high redshift (e.g., Nobuta et al. 2012; Suh et al. 2015). However, super-Eddington accretion periods are thought to be possible (Zubovas \& King 2013) and may even be required to explain the fast growth of the first SMBHs (see, e.g., Volonteri 2012, for a review); superEddington episodes could in fact be the transient phenomena in which SMBHs gain most of their mass (King 2003).

As implied by its rapid variability, the X-ray emission is produced in the inner regions surrounding the SMBH (few to tens of gravitational radii, $r_{\mathrm{G}}$; e.g., Chartas et al. 2009; Zoghbi et al. 2012; Reis \& Miller 2013) and is the most direct probe for investigating accretion properties. There are several key observables in the X-ray spectra of active galactic nuclei (AGN) related to the accretion rate. First, a positive correlation between spectral slope $(\Gamma)$ and flux (and hence $\lambda_{\text {Edd }}$ ) is thought to be a common feature of SMBH accretion and has been observed in individual, highly variable AGN at low redshift (e.g., Perola et al. 1986; Vaughan \& Edelson 2001); in large samples of AGN, both locally and at high redshift (Shemmer et al. 2008; Risaliti et al. 2009; Brightman et al. 2013); and also derived theoretically for BHs in general (Laurent \& Titarchuk 2011), with sources close to the Eddington limit showing softer spectra ( $\Gamma$ up to 2.5 ) compared to sources accreting at slower rates $(\Gamma \sim 1.5)$. There is also evidence that this relation tends to flatten above $\lambda_{\text {Edd }}=1$ (Shih et al. 2002; Ai et al. 2011). The relation between $\Gamma$ and $\lambda_{\text {Edd }}$ is based on the existence of a strong link between the accretion flow and the properties of both the disk and the corona: a high accretion rate drives the disk temperature up, producing more soft X-ray radiation and at the same time increases the Compton cooling of the corona, steepening the slope of the X-ray continuum (e.g., Shemmer et al. 2006).

Second, an anti-correlation has been observed between $\lambda_{\text {Edd }}$ and the equivalent width (EW) of the narrow component of the Fe $\mathrm{K} \alpha$ emission line $\left(E W \propto \lambda_{\mathrm{Edd}}{ }^{-0.19}\right.$; Bianchi et al. 2007): at $\lambda_{\text {Edd }}=1$ the line can be as weak as $E W=25 \mathrm{eV}$. This correlation is thought to be the fundamental cause underlying the observed X-ray Baldwin effect (Iwasawa \& Taniguchi 1993), in which the EW anti-correlates with the X-ray luminosity. The nature of this correlation and the role of selection effects in this context, however, are still debated (see Shu et al. 2012).

The $\Gamma-\lambda_{\text {Edd }}$ and $E W-\lambda_{\text {Edd }}$ relations may be related; objects with higher values of $\lambda_{\text {Edd }}$ have a steeper continuum, implying that the number of photons that can produce the fluorescent $\mathrm{Fe} \mathrm{K} \alpha$ emission is smaller, leading to a smaller EW (Ricci et al. 2013). The intensity of the fluorescent emission line and the Compton hump are also related since for a given reflector geometry, ionization parameter, and Fe abundance the relative strength of the $\mathrm{Fe} \mathrm{K} \alpha$ line and the reflected continuum is fixed (e.g., George \& Fabian 1991); therefore, in highly accreting sources the last component should also be weaker. Furthermore, AGN with high $\lambda_{\text {Edd }}$, both at low and high redshift, tend to have stronger ionized $\mathrm{Fe} \mathrm{K} \alpha$ emission at $6.9 \mathrm{keV}$, with respect to the neutral Fe K $\alpha$ line at $6.4 \mathrm{keV}$ (Nandra et al. 1996; Iwasawa et al. 2012). The same is observed in narrow-line Seyfert 1 galaxies (e.g., Comastri et al. 1998) that are also thought to have high $\lambda_{\text {Edd }}$. This is probably due to the fact that the inner disc is more strongly ionized when the central source is brighter (see, e.g., Keek \& Ballantyne 2016).

Finally, gas outflows are naturally expected during superEddington accretion phases (Zubovas \& King 2012) owing to the intense radiation pressure associated with these events. Such winds are one of the main candidates thought to produce the AGN "feedback" and to fine-tune the $M_{\mathrm{BH}}-\sigma$ relation observed in the local Universe between quiescent SMBHs and their host bulges (see Kormendy \& Ho 2013, for a review). These outflows are observed as blueshifted absorption features produced by ionized gas in optical/UV spectra of $\sim 20 \%$ of optically selected quasars (e.g., Weymann et al. 1979; Gibson et al. 2009), and are also observed in 30-40\% of X-ray spectra of local AGN (Tombesi et al. 2010) and in a few high redshift QSOs (Chartas et al. 2003; Saez et al. 2011; Lanzuisi et al. 2012; Vignali et al. 2015) in the form of highly ionized Fe absorption lines. These absorbers may be part of a single large-scale, stratified outflow observed at different locations from the black hole and spanning several orders of magnitude in ionization, column density, and velocity (Tombesi et al. 2013). Considering the few QSOs at low redshift that show strong signatures of massive winds and have reliable $\mathrm{BH}$ mass measurements, all appear to be accreting close to their Eddington limit: PG1211+143, PG0844+349, PDS 456, MCG-6-30-15 (Pounds et al. 2003a,b; Reeves et al. 2003).

The luminous QSO PG1247+267 (hereafter PG1247) at $z=$ 2.048 is candidate to be an extreme example of super-Eddington accretion, and in this paper we present new NUSTAR observations obtained with the aim of characterizing its high-energy emission. The source properties are summarized in Sect. 2. The existing XMM-Newton data (Sect. 3) are combined with the hard X-ray data from NuSTAR (Sect. 4) to constrain the highenergy properties of this extreme QSO (Sect. 5). Results from the broad-band X-ray spectral fits are summarized and discussed in Sect. 6. Throughout the paper, a standard $\Lambda-\mathrm{CDM}$ cosmology with $H_{0}=70 \mathrm{~km} \mathrm{~s}^{-1} \mathrm{Mpc}^{-1}, \Omega_{\Lambda}=0.7$, and $\Omega_{\mathrm{M}}=0.3$ is used. Errors are quoted at the $90 \%$ confidence level for one parameter of interest.

\section{Source properties}

Having $L_{\text {bol }}=1.5 \times 10^{48} \mathrm{erg} \mathrm{s}^{-1}$, an absolute magnitude of $M_{i} \sim-30$, and an observed magnitude of $i=15.37$, PG1247 is the most luminous radio-quiet ${ }^{1} \mathrm{QSO}$ at $z \sim 2 \pm 0.2$ in the SDSS DR7 (Abazajian et al. 2009; Shen et al. 2011) ${ }^{2}$. PG1247 is also the second most luminous AGN with a reverberation mapping (RM) BH mass measurement available, obtained from a spectrophotometric monitoring campaign with the $1.82 \mathrm{~m}$ Copernicus Telescope at Asiago (Trevese et al. 2007), after the lensed quasar APM 08279+5255 at $z \sim 4$ (Saturni et al. 2016). The BH mass estimate from CIII] and CIV RM is $M_{\mathrm{BH}}=8.3_{-2.7}^{+3.4} \times 10^{8} M_{\odot}$ (Trevese et al. 2014). The average $L_{\text {bol }}$ obtained from the optical continuum (between $2500 \AA$ and $5100 \AA$ ) measured in these observations is $1.1 \times 10^{48} \mathrm{erg} \mathrm{s}^{-1}$. These values translate into a fiducial value of $\lambda_{\mathrm{Edd}}=10.6$, the highest reported in the literature to date for a QSO with a SMBH mass obtained via RM.

\footnotetext{
The source is undetected (at $5 \sigma$ c.l.) in the FIRST radio survey at $1.4 \mathrm{GHz}$, and has a radio loudness $R<0.3$ (defined as $R=$ $\left.f_{1.4 \mathrm{GHz}} / f_{4400 \AA}\right)$.

${ }_{2}$ The second considering SDSS $1521+5202$ at $z=2.208$ with $M_{i} \sim-30.6$, Luo et al. (2015).
} 
The source also has a single epoch (SE) BH mass estimate, reported in Shen et al. (2011), from MgII in the range $\log \left(M_{\mathrm{BH}} / M_{\odot}\right)=9.7-9.9$, depending on the calibration adopted, and $\log \left(M_{\mathrm{BH}} / M_{\odot}\right) \sim 9.8$ from CIV. The authors take the latter as the $M_{\mathrm{BH}}$ fiducial value for all the sources with $z>1.9$ because of the SDSS observing band limits.

Although the statistical errors on these measurements are small $\left(\Delta M_{\mathrm{BH}}=0.01-0.03\right)$ one must bear in mind that there are large systematic uncertainties $(\gtrsim 0.4 \mathrm{dex})$ associated with these estimates. In particular, SE estimates based on CIV are known to be affected by large uncertainties due to the presence of outflowing components contributing to the CIV line flux (see, e.g., the discussion in Shen et al. 2011). The strong blue wing in the CIV emission line and the presence of absorption systems observed in PG1247 SDSS spectrum support this possibility. In particular, a doublet absorption line at $\sim 1508 \AA$ with a rest-frame separation of $2.57 \AA, F W H M \sim 200 \mathrm{~km} \mathrm{~s}^{-1}$ and EW doublet ratio of $\sim 0.8$ identify the 1548 and 1550 transitions of the CIV doublet line (see, e.g., Vestergaard 2003). The doublet is at $\sim-7700 \mathrm{~km} \mathrm{~s}^{-1}$ from the systemic and therefore can be associated with outflowing gas.

On the other hand, the RM measurements based on the same emission line are less affected by non-virial outflows if the root mean square spectrum (Peterson et al. 1998) is used instead of the average spectrum, because the outflow components are expected to vary on timescales longer than reverberation time lags (see the discussion in Trevese et al. 2014). In all cases, even taking into account the SE value, the source has an accretion rate in the range $\lambda_{\text {Edd }}=0.9-1.75$, which is close to or above the Eddington limit. All these properties are summarized in Fig. 1 (top), where the $L_{\mathrm{bol}}$ and $M_{\mathrm{BH}}$ values for PG1247 from Trevese et al. (2014) and from Shen et al. (2011) are shown in comparison with the $1.8<z<2.2$ quasars from the SDSS DR7 cata$\log$. PG1247 is a truly remarkable source that can be used as a laboratory for testing predictions of super-Eddington accretion theories.

A magnification factor of $\mu=5-10$ due to strong lensing could bring the Eddington ratio of PG1247 to $\sim 1$ (see discussion in Trevese et al. 2014). However, the quasar was observed several times between 1991 and 1998 with HST as a single point source, with no sign of multiple images, down to scales of $\sim 0.1^{\prime \prime}$. Figure 1 (bottom) shows a $4^{\prime \prime} \times 4^{\prime \prime}$ zoom around PG1247 from the combined HST-NICMOS observations of 1998 (F160W filter, total exposure time 1150s). The possibility of strong lensing is therefore excluded, as all known high-redshift lensed QSOs observed so far with HST have been resolved into multiple images at those scales ${ }^{3}$.

\section{Existing X-ray data}

PG1247 was observed by XMM-Newton in 2003 for a total of $34 \mathrm{ks}$ (20 ks after background-flare subtraction). No significant variability is detected within the XMM-Newton observation. Page et al. (2004) analyzed the data, and reported a strong reflection component $(R \sim 2.8$, modeled with PEXRAV, Magdziarz \& Zdziarski 1995$)$, a very soft power law $(\Gamma=2.23 \pm 0.10)$, plus a broad and neutral Fe $\mathrm{K} \alpha$ line $(\sigma=0.52 \pm 0.35 \mathrm{keV}, E W=$ $421 \pm 215 \mathrm{eV})$. As the authors pointed out, such a high reflection fraction is unphysical in the context of the PEXRAV model, which includes a primary power law and models reflection from

\footnotetext{
3 See, e.g., the CASTLES database of HST gravitational lenses (https://www.cfa.harvard.edu/castles/) observed with comparable exposure times.
}
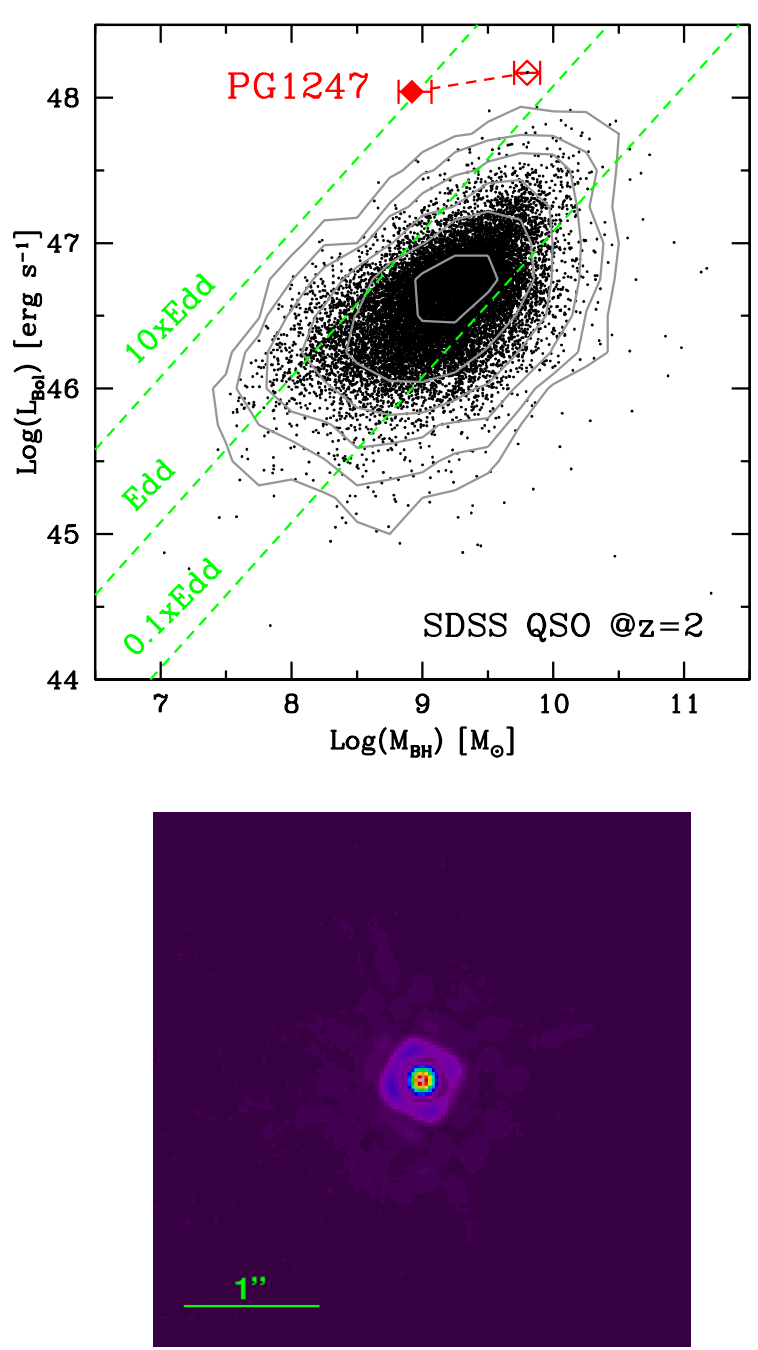

Fig. 1. Top: $L_{\mathrm{bol}}$ and $M_{\mathrm{BH}}$ for the $1.8<z<2.2$ SDSS QSO sample (black points and gray contours). Values for PG1247 from SDSS (Shen et al. 2011) and from Trevese et al. (2014) are shown with empty and filled red diamonds, respectively. Dashed green lines mark different $\lambda_{\text {Edd }}$ values. Bottom: $4^{\prime \prime} \times 4^{\prime \prime}$ HST-NICMOS image of PG1247 (filter $F 160 W$ ). The $1^{\prime \prime}$ scale is shown.

cold material in a slab geometry, since $R$ is defined to be $\Omega / 2 \pi$, i.e., the solid angle of the cold material visible from the Comptonizing source in units of $2 \pi$, and therefore $R \sim 2.8>4 \pi \mathrm{sr}$, the solid angle of the entire sphere.

We reanalyzed the data applying standard extraction procedures using the Scientific Analysis System (SAS) v13.5, and optimized extraction regions (40" for pn and $35^{\prime \prime}$ for MOS 1 and 2) to obtain pn and MOS $1+2$ spectra $^{4}$. The extracted spectra have $\sim 4560$ pn and $\sim 2900$ MOS1+2 counts in the $0.3-10 \mathrm{keV}$ band (black and red data points in Fig. 2, top). We fitted the spectrum with a single power law plus Galactic absorption $\left(N_{\mathrm{H}}^{\mathrm{Gal}}=0.9 \times 10^{20} \mathrm{~cm}^{-2}\right.$, Kalberla et al. 2005) in the observed $0.3-2 \mathrm{keV}$ band, corresponding to the rest frame $1-6 \mathrm{keV}$ band at the source redshift. Indeed, extrapolating this model to the full 0.3-10 keV observed band, strong residuals can be seen around the expected position of the $\mathrm{Fe} \mathrm{K} \alpha$ emission line, and at the reflection hump. We fitted the data with the same PEXRAV reflection model adopted in Page et al. (2004) to check for consistency. The best fit $\left(\chi^{2} /\right.$ d.o.f. $\left.=278.5 / 300\right)$ is obtained with a very soft

\footnotetext{
4 We verified that the source is too faint to have useful RGS data.
} 

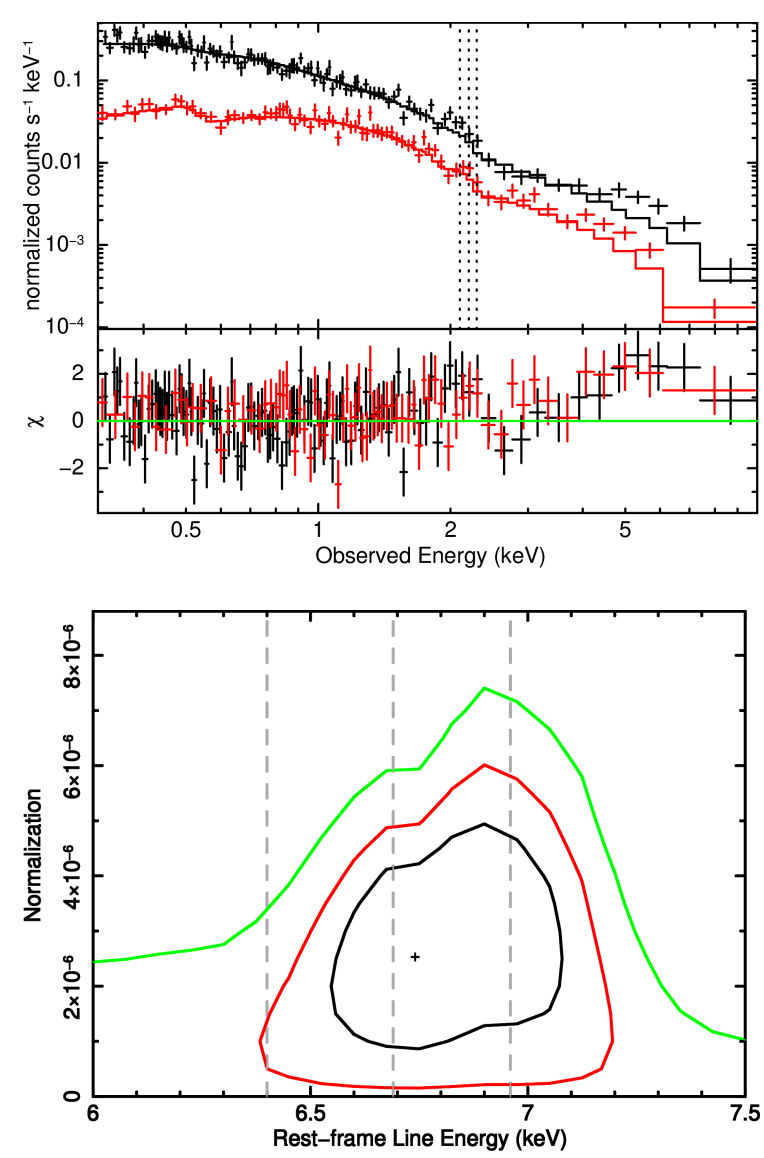

Fig. 2. Top: $X M M-N e w t o n$ pn (black) and MOS1+2 (red) spectra of PG1247. The model is a simple power law fitted to the $0.3-2 \mathrm{keV}$ observed band to highlight the residuals above $2 \mathrm{keV}$ due to the emission line complex and the strong reflection component. Bottom: contour plot (at 68, 90, and 99\% c.1.) for the emission line energy (rest frame) and normalization. In both panels the dashed lines mark the expected neutral $\mathrm{Fe}, \mathrm{FeXXV}$, and FeXXVI K $\alpha$ emission line energies.

power law with $\Gamma=2.36 \pm 0.08$, again with an extremely high reflection component $R=3.92_{-125}^{+1.80}$. The high-energy cutoff $\left(E_{\text {cut }}\right)$ is fixed to $100 \mathrm{keV}$ in the $X M M-N e w t o n$ data, given that the $X M M-N e w t o n$ spectra only reach $\sim 30 \mathrm{keV}$ (rest frame), and are therefore not able to constrain this parameter, given the available photon statistics. Also, the inclination angle (defined as the inclination angle between the line of sight and the vertical axis of the accretion disk) is fixed to the default value, $\cos (i)=0.45$ (see Sect. 5.1.1. for the effect of possible different geometries). The normalizations of pn and MOS1+2 are left free to vary independently, but they are in agreement within $\sim 4 \%$.

Residuals to the PEXRAV model in both the pn and MOS $1+2$ spectra around $6.4 \mathrm{keV}$ rest frame suggest the presence of an emission line close to the rest-frame energies of the $\mathrm{Fe} \mathrm{K} \alpha$ line. We added a narrow ( $\sigma=10 \mathrm{eV}$ fixed) emission line to the model at energy $E_{\text {line }}=6.4 \mathrm{keV}$, fixed. The improvement in the fit is small $\left(\Delta \chi^{2}=1.9\right)$ and the addition of the emission line is justified only at a $\sim 85 \%$ confidence level (c.l., F-test probability $p=0.15)^{5}$. Leaving the energy of the line as a free parameter, we obtain a further improvement of the fit of

5 The F-test is known to yield only an approximate probability of finding an emission line at a given energy, and only if the line energy is known in advance (see, e.g., Protassov et al. 2002; Markowitz et al. 2006).
$\Delta \chi^{2}=3.0$. The addition of the new free parameter is justified at $\sim 93 \%$ c.l. ( $p=0.071$ from an F-test). The best-fit line energy is $6.78_{-0.32}^{+0.42} \mathrm{keV}$. Figure 2 (bottom) shows the confidence contours of the emission-line energy and normalization, obtained for a fixed $\sigma=10 \mathrm{eV}$. As can be seen in the contours, the observed feature could be the result of blending of Fe XXV and Fe XXVI, while the $6.4 \mathrm{keV}$ energy is ruled out at $90 \%$ c.l. Leaving the line width $\sigma$ as a free parameter, the best-fit values become $E_{\text {line }}=6.82_{-0.30}^{+0.42} \mathrm{keV}$ and $\sigma \leq 0.63 \mathrm{keV}$. The improvement in the fit is minimal $\left(\chi^{2} /\right.$ d.o.f. $\left.=272.6 / 297\right)$ and the new free parameter is not justified $(p=0.30)$. As pointed out in Page et al. (2004), although there is evidence that the iron line may be broadened, no definite statement can be made. A narrow emission line with the same parameters derived above is included in all the following fits. The equivalent width of the line is $E W=168_{-150}^{+198} \mathrm{eV}$ rest frame. Given the inferred luminosity of $L_{2-10}=10^{46} \mathrm{erg} \mathrm{s}^{-1}$, the X-ray Baldwin effect predicts $E W=20-30 \mathrm{eV}$ (Bianchi et al. 2007). Assuming that this value refers to a canonical, $R=1$ reflection component with solar abundances, the observed emission line EW is broadly consistent with the strong reflection component $(R=3-4)$ found above.

PG1247 was also observed by Swift-XRT 19 times between 2007 and 2014. We collected the spectra in all the observations with more than $1 \mathrm{ks}$ exposure time (14 in total), and merged them using standard ftool procedures to obtain a single spectrum with statistics good enough to perform a basic spectral analysis. The final spectrum has $\sim 560$ net counts in the $0.3-10 \mathrm{keV}$ band for a total exposure time of $43 \mathrm{ks}$. When fitted with the same reflection model described above, the XRT data give consistent results with the XMM-Newton spectra: a steep power law $(\Gamma=$ $2.2 \pm 0.4)$ and a strong reflection component $(R \sim 2.5)$. Given the limited number of counts, and the fact that they have been collected over 7 years, we decided not to use the XRT spectrum for the following spectral analysis. However, we used this bestfit model to convert the observed count rate of each observation into full band $(0.2-10 \mathrm{keV})$ fluxes, which will be relevant for the variability analysis of the source (see Sect. 35.2 and Fig. 7).

\section{NuSTAR data}

PG1247 was observed by NuSTAR (Harrison et al. 2013) in December 2014 for a total of $94 \mathrm{ks}$. The raw data were processed using the NuSTAR Data Analysis Software package v. 1.4.1 $(\text { NuSTARDAS })^{6}$. Calibrated and cleaned event files were produced using the calibration files in the NuSTAR CALDB (version 20150312) and standard filtering criteria with the nupipeline task. The global light-curves extracted from the full FPMA and FPMB modules in the energy range 3-20 keV show a strong background flare - with a count-rate more than a factor of two higher than the stable level - due to solar activity, lasting $\sim 2 \mathrm{~h}$. We removed this high background period using the nustar_filter_lightcurve IDL script. The cleaned exposure time is $86 \mathrm{ks}$.

The NUSTAR observation of PG1247 is also affected by contamination (particularly in FPMA) by the Coma cluster, lying $\sim 2.5^{\circ}$ northeast of the quasar. Figure 3 (top), shows the 3-79 keV images of PG1247 obtained with the FPMA (left) and FPMB (right) detectors produced with the IDL code nuskybkg (Wik et al. 2014). This code takes into account all the background components usually observed in NuSTAR, i.e., the

\footnotetext{
6 http://heasarc.gsfc.nasa.gov/docs/nustar/analysis/ nustar_swguide.pdf
} 

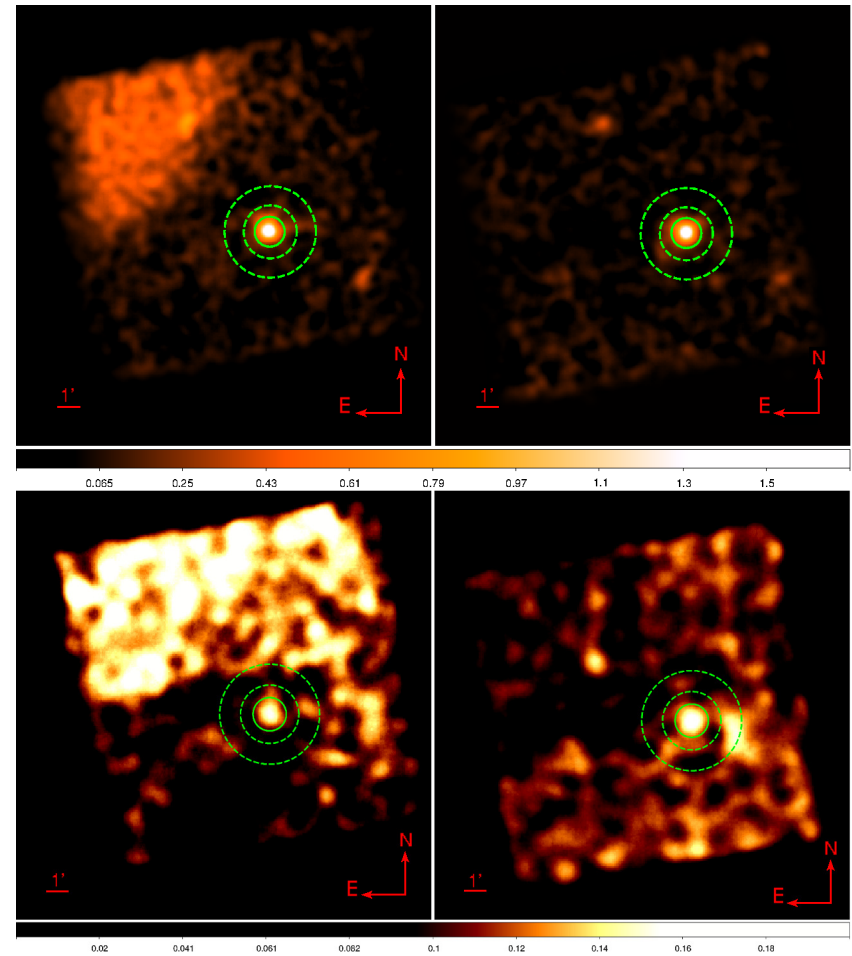

Fig. 3. Top: 3-79 keV background-subtracted images of the NuSTAR observation of PG1247 (FPMA left and FPMB right). Contamination from the Coma cluster is visible in the upper left corner of FPMA. Bottom: 15-30 keV NuSTAR images, not background subtracted. In both panels the $1^{\prime}$ scale is shown, and the source and background extraction regions are shown with continuous and dashed lines, respectively. North is up and east is left.

instrumental background, the focused cosmic X-ray background, and the aperture background. However, the code does not model and remove the contamination from the nearby cluster, which is evident in the upper left corner of the FPMA image. Therefore, we used annular regions to extract local background spectra instead of using the background files produced by nuskybkg. The background spectra were extracted from annular regions of inner radius $70^{\prime \prime}$ and outer radius $120^{\prime \prime}$. Circular extraction regions for the source, with different radii, were tested in order to find the radius that optimizes the $\mathrm{S} / \mathrm{N}$ for each focal plane module. The final extraction radius is $40^{\prime \prime}$ for both FPMA and FPMB, which gives a S/N of $\sim 14$ and $\sim 15$, respectively. The nuproducts task was then used to extract the NuSTAR source and background spectra, plus the appropriate response and ancillary files. In the following the fits are performed leaving the relative normalizations between FPMA and FPMB as free parameters. The two normalizations agree within $15 \%$ and are consistent within $1 \sigma$.

The spectra have been grouped using the tool Specgroup within SAS, to a minimum $S / N=3$ per bin. The source is detected at this level between 3 and $30 \mathrm{keV}$ in both instruments, with a total of 520 (590) net counts for FPMA (FPMB). Figure 3 (bottom) shows the 15-30 keV NuSTAR image of PG1247 without any background subtraction: the source is clearly detected even in this very hard band. Figure 4 shows the NuSTAR spectra (FPMA in cyan and FPMB in blue) fitted with a simple power law $\left(\Gamma=2.14 \pm 0.13, \chi^{2} /\right.$ d.o.f. $\left.=65.46 / 73\right)$. The curved shape of the hard X-ray spectrum of PG1247 is visible in the residuals (central panel). Adopting a PEXRAV model, the fit is significantly improved $\left(\chi^{2} /\right.$ d.o.f. $\left.=55.83 / 71\right)$. Residuals for this fit are shown in the lower panel of Fig. 4. However, owing to the

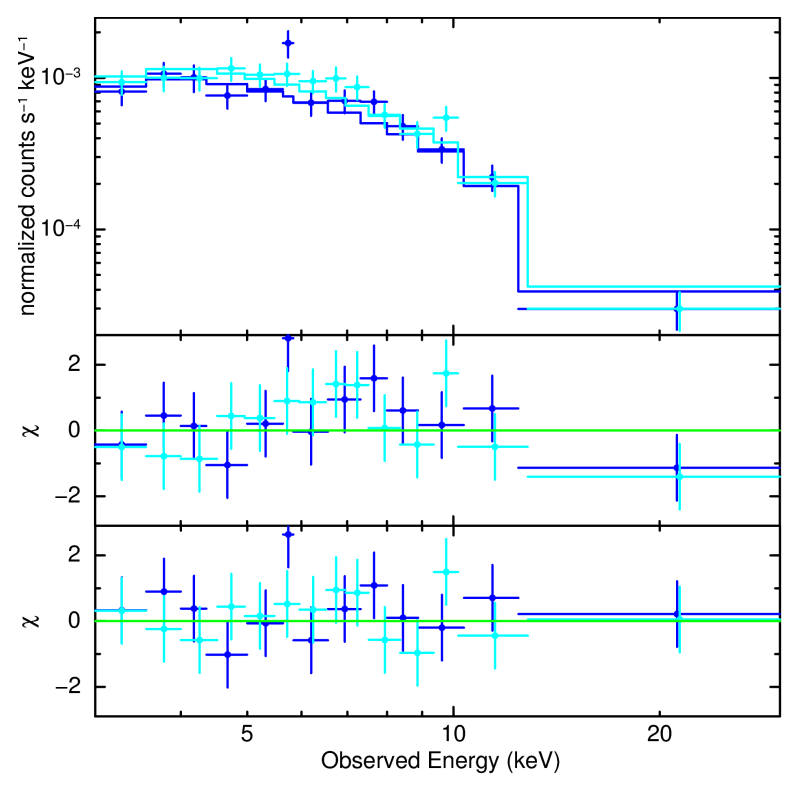

Fig. 4. NUSTAR FPMA and FPMB data (blue and cyan points, respectively) fitted with a simple power law (top panel). The spectra have been further rebinned with respect to the $S / N=3$ level to highlight the curvature of the spectrum in the residuals (central panel). The residuals with respect to the fit performed with the PEXRAV model are shown in the lower panel.

limited photon statistics and to the complexity of the underlying model, the NUSTAR spectra alone do not provide interesting simultaneous constraints on the high-energy cutoff, the reflection fraction, and the photon index; the best-fit photon index is $\Gamma=1.9 \pm 1.5$, while the high-energy cutoff and reflection fraction are only loosely constrained $\left(E_{\text {cut }}>50 \mathrm{keV}, R>1.35\right)$.

The flux in the $2-10 \mathrm{keV}$ band (extrapolated using the PEXRAV model) is $F_{2-10}=(4.2 \pm 0.5) \times 10^{-13} \mathrm{erg} \mathrm{cm}^{-2} \mathrm{~s}^{-1}$, while the $2-10 \mathrm{keV}$ flux measured by XMM-Newton with the same model was $F_{2-10}=(2.3 \pm 0.1) \times 10^{-13} \mathrm{erg} \mathrm{cm}^{-2} \mathrm{~s}^{-1}$ : the $N U S T A R$ data show that the source has increased in flux between the XMM-Newton observation in 2003 and the NuSTAR observation in 2014 by a factor of $\sim 1.8$. Also, the intermediate SwiftXRT observations show an average flux level consistent with the NUSTAR measurement. The cross-calibration between NuSTAR and the other X-ray satellites is accurate within $10 \%$ (Madsen et al. 2015).

Because the correlation between $\Gamma$ and flux (i.e., $\lambda_{\text {Edd }}$ ) saturates at high fluxes (e.g., Shih et al. 2002; Ai et al. 2011), we try to fit the NuSTAR spectra with $\Gamma$ fixed at the XMM-Newton value to get more stringent constraints on the $E_{\text {cut }}$ and $R$ parameters, and test whether there is any difference, for example, in the $R$ value derived from $X M M-N$ ewton due to the change in flux (i.e., accretion rate). The resulting best fit $E_{\text {cut }}$ is $E_{\text {cut }}>60 \mathrm{keV}$, while the reflection fraction is $R=2.7_{-1.4}^{+3.4}$, and therefore consistent with the XMM-Newton results within the large errors.

\section{Joint spectral modeling}

We modeled the XMM-Newton and NUSTAR spectra together with the aim of deriving a physically meaningful interpretation of the peculiar X-ray spectrum of PG1247, leveraging the broad band covered by the combined data and accounting for the higher flux measured during the NUSTAR observation. First, we adopted a cold reflection model and tested whether the addition of the new NUSTAR data allow for a less extreme value of 

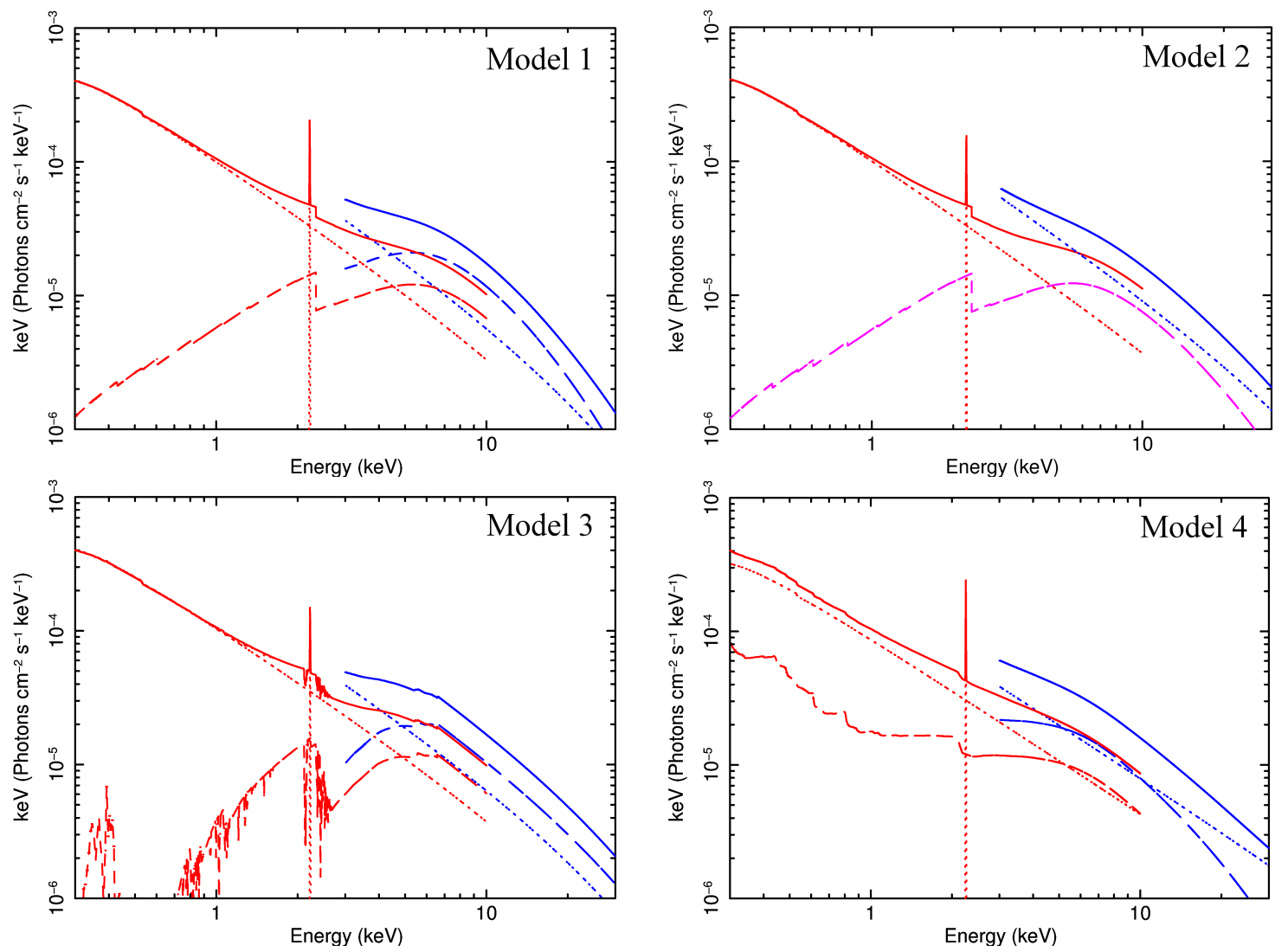

Fig. 5. Best-fit unfolded models for models 1, 2, 3, and 4, from top left to bottom right. The continuous curves represents the best fit to the $X M M-N e w t o n$ data (in red) and NUSTAR data (in blue). The dotted curves represent the primary power law, while the dashed curves represent the component reproducing the hump (either reflection or absorption). In the top right panel the magenta curve represents the constant reflection component underlying both XMM-Newton and NUSTAR data, modeled with PEXRAV.

$R$ (Sect. 5.1). Then we tested whether the strong reflection seen in XMM-Newton can be explained in terms of variability of the continuum superimposed on a constant cold reflection component (Sect. 5.2). Third, we tested the possibility that the hump observed in the hard X-rays is produced by absorption of partial covering, dense material, rather than by reflection (Sect. 5.3). Finally we tested a model in which the reflection is produced in the inner regions of an ionized accretion disk, and is relativistically blurred (Sect. 5.4).

\subsection{Variable cold reflection}

To account for the overall variability of the X-ray spectra between 2003 and 2014, we modeled the XMM-Newton+NuSTAR data with a cold reflection model (PEXRAV) as described in Sect. 3, but where the normalizations of the XMM-Newton and $N U S T A R$ data are allowed to vary independently, while the rest of the free parameters, i.e., $\Gamma, E_{\text {cut }}$, and $R$, are forced to be the same for the two data sets (model 1); i.e., we are assuming that the spectral shape was the same in the two periods, and only the global normalization has changed. The inclination angle was first fixed to $\cos (i)=0.45$.

As a first step we fixed the value of the reflection parameter to $R=1$ to check whether the addition of NuSTAR data allow for a less extreme reflection component with respect to the $X M M-N e w t o n$ data alone. The resulting fit gives $\chi^{2} /$ d.o.f. $=$ $347.8 / 369$, with a soft photon index $(\Gamma=2.22 \pm 0.05)$, while $E_{\text {cut }}$ is not well constrained. We then left the reflection parameter $R$ free to vary (model 1, Fig. 5, top left). The best-fit value for the reflection parameter is $R=3.8_{-1.5}^{+2.0}$ and the improvement in the fit is large $\left(\Delta \chi^{2}=20.1\right)$. The F-test indicates that the addition of this extra free parameter provides a significant improvement to the fit (F-test probability $\left.p=2.7 \times 10^{-6}\right)$. The best-fit photon index is again very soft $\left(\Gamma=2.35_{-0.08}^{+0.09}\right)$, comparable to the one obtained from $X M M-N e w t o n$ alone. With this model the $E_{\text {cut }}$ is constrained to a rather low value $\left(E_{\text {cut }}=89_{-34}^{+112} \mathrm{keV}\right.$ rest frame). The best-fit parameters are given in Table 1 . The unfolded data and model for the best fit obtained in this way are shown in panel a of Fig. 6. Panel b shows the $\Delta \chi$ for the same model.

We note that a small inclination angle $i$ between the line of sight and the accretion disk axis can increase the reflection component observed by at most a factor of $\sim 2$ with respect to the default geometry used in this section $(\cos (i)=0.45)$. Therefore, even an extremely face-on geometry cannot entirely account for the $R>3$ reflection observed in the data. We obtain $R=2.5_{-1.4}^{+1.4}$ for $\cos (i)=0.95$, still significantly larger than $R=1$, which is the reflection for a $2 \pi$ sr solid angle. We therefore discard this model as unrealistic.

\subsection{Constant cold reflection}

To reproduce a case in which a variable primary power law is superimposed on a constant reflection component, we used a model that includes a power law with high-energy exponential cutoff and the normalization left free to vary between the XMM-Newton and NUSTAR observations, plus a PEXRAV component seen only 
Table 1. Fit parameters for the baseline models discussed in Sect. 5.

\begin{tabular}{|c|c|}
\hline Model 1: & pexrav (var. cold refl.) \\
\hline $\begin{array}{l}\Gamma \\
E_{\text {cut }} \quad(\mathrm{keV}) \\
R \\
\chi^{2} / \text { d.o.f. }\end{array}$ & $\begin{array}{l}2.35_{-0.08}^{+0.09} \\
89_{-34}^{+112} \\
3.8_{-1.5}^{+2.1} \\
327.1 / 367\end{array}$ \\
\hline Model 2: & pexrav (const. refl.) \\
\hline $\begin{array}{l}\Gamma \\
E_{\text {cut }} \quad(\mathrm{keV}) \\
R \\
\chi^{2} / \text { d.o.f. } \\
\end{array}$ & $\begin{array}{l}2.37_{-0.08}^{+0.09} \\
163_{-83}^{+470} \\
3.8 \pm 1.4 / 1.7 \pm 0.8^{a} \\
327.7 / 367\end{array}$ \\
\hline Model 3: & pexrav $\times$ zxipcf (ion. abs.) \\
\hline $\begin{array}{l}\Gamma \\
E_{\text {cut }} \quad(\mathrm{keV}) \\
N_{\mathrm{H}} \quad\left(\mathrm{cm}^{-2}\right) \\
\mathrm{CF} \\
\log \xi \quad\left(\mathrm{erg} \mathrm{cm} \mathrm{s}^{-1}\right) \\
\chi^{2} / \text { d.o.f. }\end{array}$ & $\begin{array}{l}2.33 \pm 0.10 \\
96_{-47}^{+181} \\
1.3 \pm 0.3 \times 10^{24} \\
0.62 \pm 0.1 \\
2.4 \pm 0.4^{b} \\
325.5 / 365\end{array}$ \\
\hline Model 4: & relxill_lp (relativistic refl.) \\
\hline $\begin{array}{l}\Gamma \\
h \quad\left(r_{\mathrm{G}}\right) \\
a \\
\cos (i) \\
\log \xi \quad\left(\mathrm{erg} \mathrm{cm} \mathrm{s}^{-1}\right) \\
\chi^{2} / \text { d.o.f. }\end{array}$ & $\begin{array}{l}2.26 \pm 0.04 \\
<3.5 \\
>0.68 \\
>0.83 \\
1.8 \pm 0.5^{c} \\
333.8 / 365\end{array}$ \\
\hline
\end{tabular}

Notes. Model 1 is a PEXRAV model with all the parameters linked between XMM-Newton and NUSTAR, except for the normalizations. Model 2 has the normalization of the reflection component linked between the XMM-Newton and NUSTAR data, while the primary power law is left free to vary independently, so that the two data sets have two different $R$ parameters. The $\Gamma$ and high-energy cutoff are linked between the power-law and the reflection component, and between the two data sets. Model 3 includes an ionized absorber with partial covering superimposed on a PEXRAV model with $R=0$. Model 4 is a RELXILL_LP relativistic reflection model, where the $E_{\text {cut }}$ is frozen to $300 \mathrm{keV}$. In Models 1, 2, and 3 the inclination angle is frozen to the default value $\cos (i)=0.45 .{ }^{(a)} R$ values for XMM-Newton and NuSTAR, respectively. ${ }^{(b)}$ Ionization parameter of the obscuring material. ${ }^{(c)}$ Ionization parameter of the accretion disk.

in reflection $(R=-1)$ and kept constant between the two observations (model 2, Fig. 5 top right). The photon index and $E_{\text {cut }}$ are left as free parameters, but are forced to have the same value in the power-law and PEXRAV components. In this way, the extreme $R$ parameter observed in the XMM-Newton spectra of 2003 could be explained in terms of a primary power law observed in a lower state with respect to the reflection hump, which is expected to be less variable and to exhibit significant time lags, being the result of reflection from distant material (i.e., the torus) with a range of different light paths. The goodness of the fit in this case is comparable with that of model $1\left(\chi^{2} /\right.$ d.o.f. $\left.=327.7 / 367\right)$. The $\Delta \chi$ for this model are shown in panel c of Fig. 6 . The resulting $R$ parameters for the fit with a constant reflection component are $R=3.8 \pm 1.4$ for the XMM-Newton spectra and $R=1.7 \pm 0.8$ for the NUSTAR spectra. If this model is correct, the best-fit cutoff is $E_{\text {cut }} \sim 160 \mathrm{keV}$ (rest frame), a factor of 2 higher than the value measured for model 1 and with larger errors: it is loosely

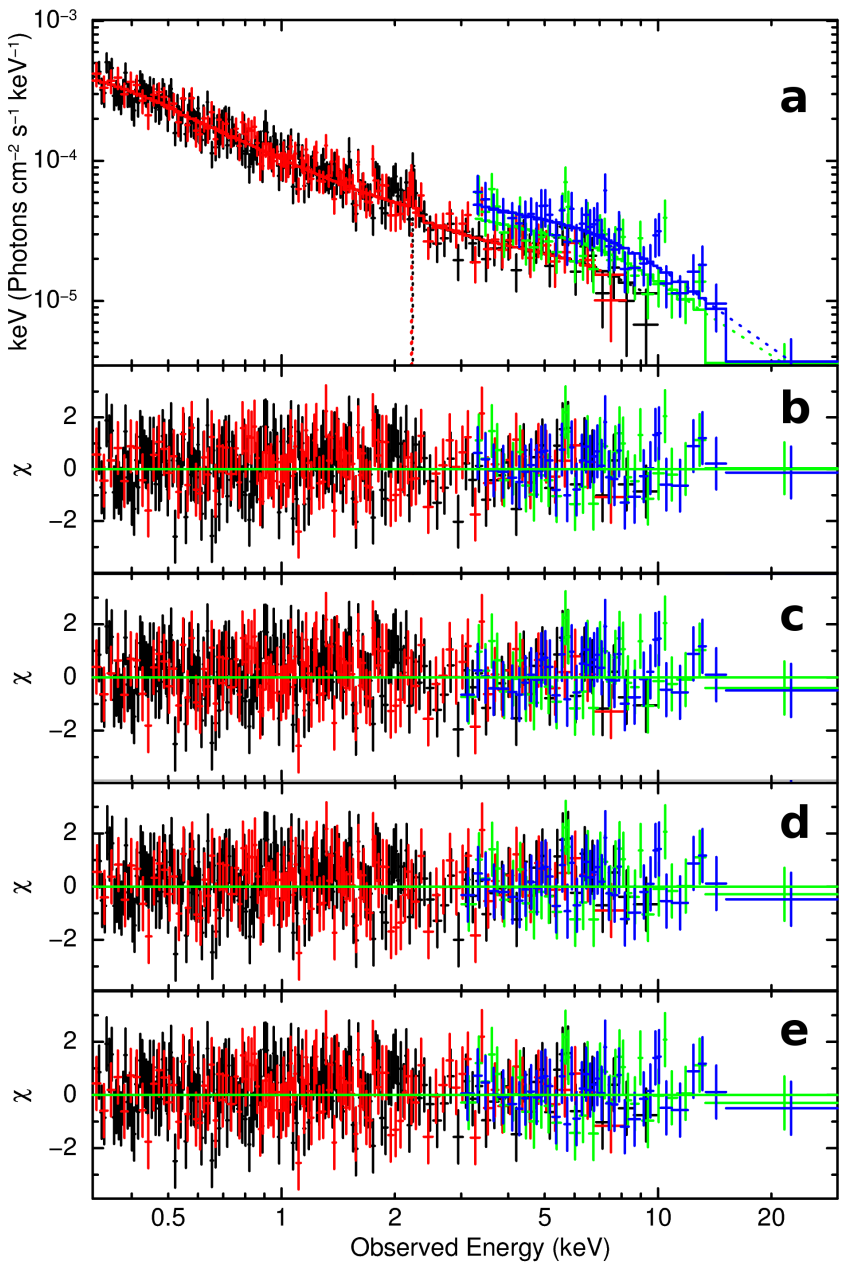

Fig. 6. Panel a) unfolded data and model for the best fit obtained with model 1. Panels b)-e) delta $\chi$ for model 1, 2, 3, and 4, respectively. In all panels $X M M-N e w t o n$ pn data are in black, MOS $1+2$ in red, NuSTAR module $\mathrm{A}$ in green, and $\mathrm{B}$ in blue.

constrained to be $E_{\text {cut }} \lesssim 600 \mathrm{keV}$ at $90 \%$ c.l. Given that the NUSTAR data only reach a $90 \mathrm{keV}$ rest frame, some doubt can be cast on the reliability of this result.

The weak Fe line EW observed in the XMM-Newton data $\left(E W=168_{-150}^{+198} \mathrm{eV}\right.$, see Sect. 3) is consistent, within the errors, with the expected $\mathrm{Fe}$ line EW computed taking into account the high reflection factor observed in that data set and the X-ray Baldwin effect $(E W \sim 20-30 \mathrm{eV}$ for the luminosity of PG1247, to be rescaled by a reflection parameter $R=3.8$ ) without requiring any particularly low $\mathrm{Fe}$ abundance.

Figure 7 shows the $0.2-10 \mathrm{keV}$ (rest-frame) light-curve of PG1247 as has been observed since 1993 by ROSAT, ASCA, XMM-Newton, and Swift. The light-curve is taken from Shemmer et al. (2014). We added four more Swift observations taken after November 2013, and as an empty square the flux obtained from the reanalysis of the ROSAT data performed in Boller et al. (2016), with a better background treatment. The NuSTAR data point (magenta) is added by extrapolating the hard $\mathrm{X}$-ray flux to the $0.2-10 \mathrm{keV}$ flux, using the spectral model described in this section. PG1247 is highly variable at X-ray wavelengths despite its exceptional X-ray luminosity: the normalized excess variance (e.g., Nandra et al. 1997) is $\sigma_{\text {rms }}^{2}=0.14 \pm 0.09$ (Shemmer et al. 2014), higher than expected for its X-ray luminosity, i.e., $\sigma_{\mathrm{rms}}^{2} \sim 0.01$ (Young et al. 2012; Lanzuisi et al. 2014). More importantly, the light-curve shows that the flux level of 


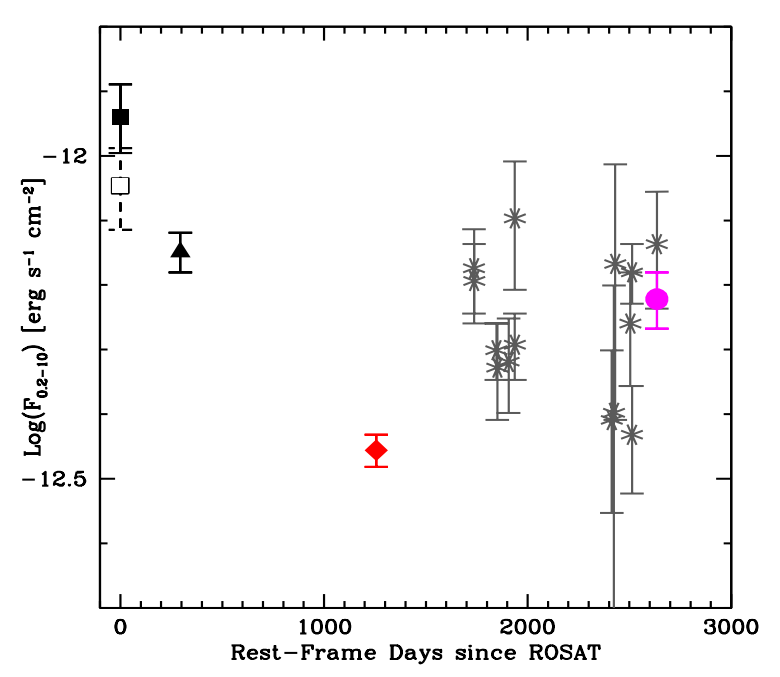

Fig. 7. Rest-frame $0.2-10 \mathrm{keV}$ light-curve of PG1247 since its first detection with ROSAT in 1993 (black filled square). The empty square shows the ROSAT data reanalyzed in Boller et al. (2016). The black triangle represents the ASCA measurement (1995). The red diamond represents the XMM-Newton measurement of 2003. The gray points represent the monitoring performed with Swift between 2007 and 2013 (Shemmer et al. 2014), and the magenta point shows the NuSTAR measurement in 2014.

the XMM-Newton observation (red point) is the lowest measured in the last twenty years: the X-ray flux dropped from $\sim 10$ to $3.5 \times 10^{-13} \mathrm{erg} \mathrm{s}^{-1} \mathrm{~cm}^{-2}$ between the ROSAT and the XMM-Newton measurements from 1993 to 2003 ( $\sim 1200$ days rest frame), while the flux measured by Swift between 2007 and 2013 spans the range $4-8 \times 10^{-13} \mathrm{erg} \mathrm{s}^{-1} \mathrm{~cm}^{-2}$. In this scenario, we obtain a lower limit of the distance between the primary $\mathrm{X}$-ray source and the reflector of $\sim 1200$ light-days, equivalent to $\sim 1 \mathrm{pc}$, which is consistent with molecular torus size estimates obtained from the source luminosity (see Sect. 6).

\subsection{Ionized partial covering absorber}

We then tested the possibility that an ionized, partial covering absorber could reproduce the shape observed in PG1247 without the need of any reflection component (or with a standard $R=1$ cold reflection) by affecting mostly the X-ray data below $10 \mathrm{keV}$ (rest frame). Model 3 (Fig. 5, bottom left) includes an ionized absorber with partial covering (ZXIPCF in Xspec ${ }^{7}$, Reeves et al. 2008), superimposed on a PEXRAV model with the reflection fraction parameter set to $R=0$ (i.e., no reflection). Indeed, this model can accurately reproduce the continuum shape observed in PG1247: the best fit gives $\chi^{2} /$ d.o.f. $=325.5 / 365$.

To fit the XMM-Newton and NuSTAR data, a mildly ionized $\left(\log (\xi) \sim 2.4 \mathrm{erg} \mathrm{cm} \mathrm{s}^{-1}\right)$, almost Compton thick $\left(N_{\mathrm{H}} \sim\right.$ $\left.1.3 \pm 0.3 \times 10^{24} \mathrm{~cm}^{-2}\right)$ absorber is required. The covering fraction $(C F)$ of the absorber is $C F \sim 0.6$. Figure 8 (left) shows the confidence contours for the column density and the covering fraction. Adding a standard reflection component with $R=1$ has the only effect of reducing the covering factor to $C F \sim 0.4$.

We note that the validity of Xstar is restricted to $N_{\mathrm{H}} \leq$ $1 \times 10^{24} \mathrm{~cm}^{-2}$ in order to avoid significant effects from Compton scattering (Kallman \& Bautista 2001), even if the nominal $N_{\mathrm{H}}$

This model is based on the Xstar photo-ionization code (Kallman \& Bautista 2001), where the ionization parameter is defined as $\xi=$ $L /\left(n * R^{2}\right), L$ is the luminosity of the ionizing source, $n$ the density of the ionized medium, and $R$ the distance between the two. range of ZXIPCF reaches $N_{\mathrm{H}}=5 \times 10^{24} \mathrm{~cm}^{-2}$. However, even imposing an $N_{\mathrm{H}}$ upper limit equal to $N_{\mathrm{H}}=1 \times 10^{24} \mathrm{~cm}^{-2}$ in our fit, does not significantly change the results for the other parameters or the quality of the final best fit.

This model implies that the intrinsic luminosity of the source is a factor of $\sim 2$ higher than the value observed by $X M M-N e w t o n$, going from $L_{2-10}=8 \times 10^{45} \mathrm{erg} \mathrm{s}^{-1}$ to $L_{2-10}=$ $1.5 \times 10^{46} \mathrm{erg} \mathrm{s}^{-1}$. This, however, has a limited effect on the bolometric luminosity of the source, which is estimated to be $L_{\mathrm{bol}}=1.5 \times 10^{48} \mathrm{erg} \mathrm{s}^{-1}$ from the optical continuum, given that at these luminosities the X-ray contribution is expected to be a small fraction of the total luminosity: the bolometric correction $k_{\text {bol }}$ (defined as $L_{\text {bol }} / L_{2-10}$ ) is $\sim 100$ already at $L_{\text {bol }}=10^{47} \mathrm{erg} \mathrm{s}^{-1}$ (Steffen et al. 2006; Lusso et al. 2012).

Interestingly, this model naturally produces absorption features at energies above the $\mathrm{Fe} \mathrm{K} \alpha$ line and could also produce significant $\mathrm{Fe} \mathrm{K} \alpha$ emission if the covering factor of the wind/outflow is large (i.e., P Cygni profile, see Nardini et al. 2015). A similar feature is indeed observed in the XMM-Newton spectrum of PG1247 (NUSTAR does not cover the energy of interest), albeit at low significance $(\sim 2 \sigma)$ and at energies slightly higher $\left(E_{\mathrm{abs}} \sim 7.8 \mathrm{keV}\right.$ rest frame $)$ than the value expected for FeXXV-FeXXVI K $\alpha$ transitions $(6.70-6.97 \mathrm{keV})$. Therefore, if produced by FeXXV-FeXXVI, the observed feature would imply an outflow velocity of $v_{\text {out }} \sim 0.15 c$, comparable to typical outflow velocities observed in local Seyferts (Tombesi et al. 2010). However, the quality of the spectrum and the low significance of the feature do not allow us to investigate in detail the properties of this potential outflow. Figure 8 (right) shows the ratio (in rest-frame energy) between the data and a simple power law in the region of the $\mathrm{Fe} \mathrm{K} \alpha$ line. We note that the absorption feature is seen at energies $E_{\mathrm{abs}} \sim 2.4-2.6 \mathrm{keV}$ observed frame, and therefore just above the prominent edge in the XMM-Newton mirror effective area at $E_{\text {edge }}=2.2-2.3 \mathrm{keV}$ due to the $\mathrm{Au} \mathrm{M}$ edge. This can produce some additional uncertainties in the instrumental calibration at these energies.

If this model is correct, we have detected for the first time the high energy cutoff of the primary continuum emission in a high redshift, non-lensed quasar (but see also Dadina et al. 2016, for the detection of a high-energy cutoff in a lensed QSO at $z=3.6$ ). The low value measured may have important implications for the electron temperature of the corona (see discussion is Sect. 6.2).

\subsection{Relativistic reflection}

Finally, to test the alternative possibility that the reflection continuum is produced in the inner regions of the accretion disk, i.e., by ionized material and under relativistic effects, we used the RELXILL model (Garcia et al. 2014), which is the convolution of the XILLVER reflection model (Garcia et al. 2010) for ionized accretion disk reflection, with the relativistic treatment of RELCONV (Dauser et al. 2010). In particular the RELXILL_LP configuration assumes a simple lamp post geometry, i.e., a pointlike hard X-ray source (the corona) above the black hole that is irradiating the accretion disk (e.g., Matt et al. 1991; Dauser et al. 2013) and computes both the expected emissivity profile and the strength of the reflection self-consistently (model 4, Fig. 5 bottom right).

The free parameters, in addition to the primary power-law photon index, high-energy cutoff, and normalizations, are the height of the primary source $(h)$, the BH spin $(a)$, the inclination angle of the disk $(i)$, and the ionization parameter $(\xi)$, defined as the ionization parameter of the accretion disk. The inner 

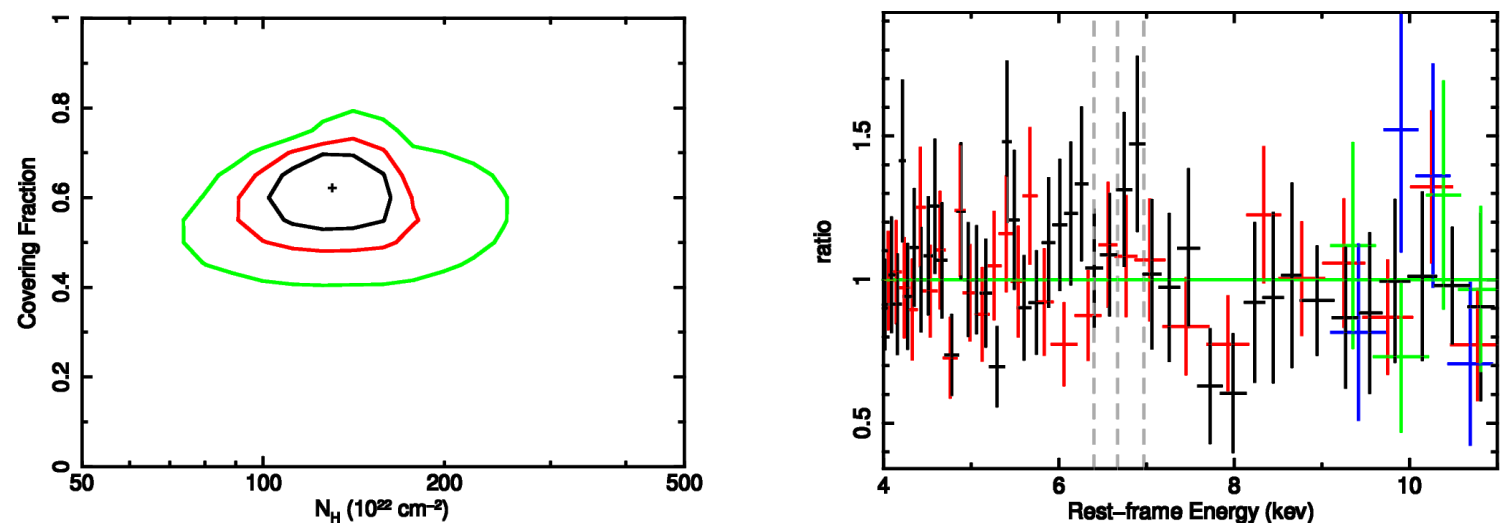

Fig. 8. Left: contour plot of the column density vs. covering fraction for model 3. Right: rest-frame residuals in the Fe K $\alpha$ line region, after fitting with a simple power law. The gray dashed lines mark the expected neutral Fe, FeXXV, and FeXXVI K $\alpha$ emission line energies. Symbols as in Fig. 6.
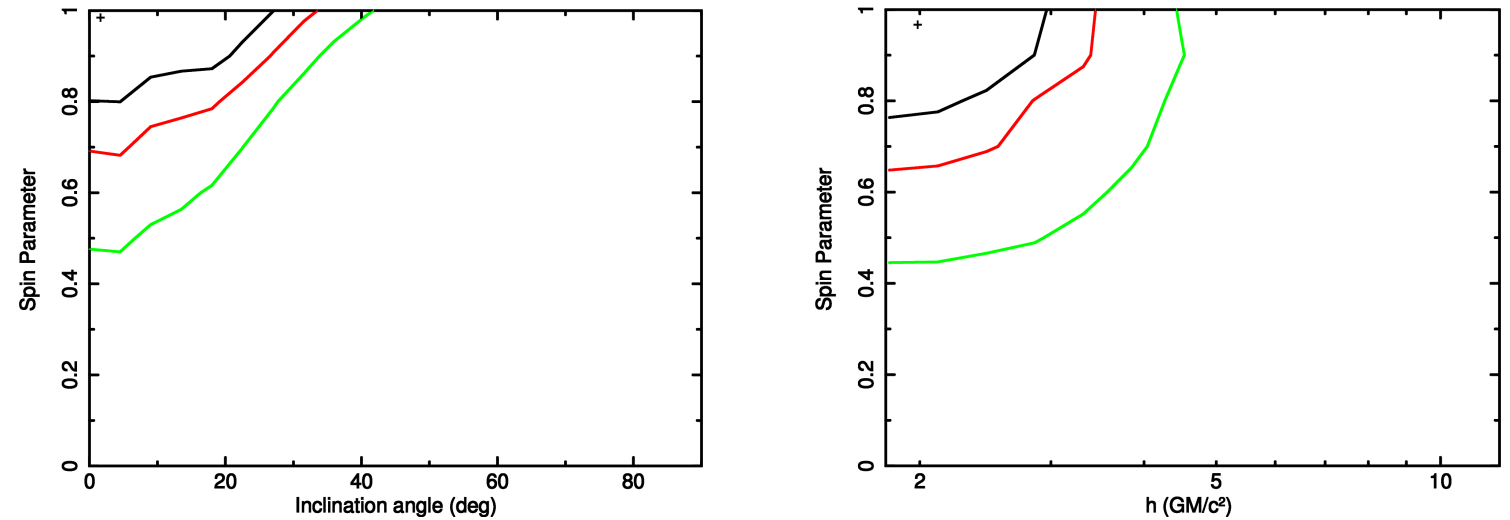

Fig. 9. Left: contour plot of the disk inclination angle vs. BH spin parameter for model 4. Right: contour plot of the corona height $h$ vs. spin parameter for model 4.

radius of the accretion disk is fixed to the innermost stable circular orbit, which depends on the $\mathrm{BH}$ spin, while the outer radius is frozen to $400 r_{\mathrm{G}}$.

Interestingly the high-energy cutoff seems to be unconstrained for this model. This is not entirely due to the limited spectral quality of PG1247 and the larger number of free parameters for this model, but also to the fact that - for a given primary power-law cutoff - the reflection component computed by RELXILL always has a sharper decline at high energies than the value computed by PEXRAV (see, e.g., Dauser et al. 2016). This means that the relativistic reflection model is able to reproduce the shape of the high-energy spectrum without the need of a cutoff. Therefore, we fixed it to $E_{\text {cut }}=100 \mathrm{keV}$, in order to be consistent with the measurements of the other parameters performed from the models discussed above. The goodness of this fit is comparable with the fits obtained for the previous three models $\left(\chi^{2} /\right.$ d.o.f. $\left.=333.8 / 365\right)$. The photon index is very soft $(\Gamma=2.26 \pm 0.04)$ also for this model.

The interesting aspect of this model is that the reflection fraction in this configuration is not a free parameter, but instead the reflection contribution is computed self-consistently as the result of the geometry, size, and ionization state of the emitting corona. The height of the corona, the $\mathrm{BH}$ spin, and the reflection fraction are indeed correlated in this model, in the sense that in order to produce a strong reflection fraction, both a low value of $h$ (i.e., the corona is close to the $\mathrm{BH}$ and hence to the disk) and a high value of the $\mathrm{BH}$ spin (i.e., the disk has a small inner radius) are required (see Dauser et al. 2014). Figure 9 (left) shows the inclination angle vs. $\mathrm{BH}$ spin parameter confidence contours: to produce the strong reflection we see in the spectrum of PG1247, a nearly maximally rotating $\mathrm{BH}$ is required $(a>0.68)$, observed at a small inclination angle $(\cos (i)>0.83)$. Figure 9 (right) shows the confidence contours between the coronal height $h$ (in units of $r_{\mathrm{G}}$ ) and the spin parameter. The height of the emitting region is constrained to be $h \leq 3.5 r_{\mathrm{G}}$. Finally, the ionization parameter is constrained to be $\log (\xi)=1.8 \pm 0.5$ erg $\mathrm{cm} \mathrm{s}^{-1}$; i.e., the disk must be moderately ionized in order to produce the strong Compton hump, while a more ionized reflecting medium would produce a steeper reflection continuum. We note that a small height of the corona $\left(h<10 r_{\mathrm{G}}\right)$ for a maximally rotating $\mathrm{BH}(a>0.9)$, observed at small inclination angles $\left(i<30^{\circ}\right)$, has also been estimated in a few local Seyferts through spectral-timing analysis and reverberation techniques (e.g., Cackett et al. 2014; see Uttley et al. 2014, for a review), while evidence is mounting that maximally rotating SMBHs may be common among AGN at low and high redshift (Walton et al. 2013; Reis et al. 2014; Reynolds et al. 2014), with the caveat that high spin means high accretion efficiency (Vasudevan et al. 2016) so the brightest objects (for which measuring the BH spin is feasible) in a population of objects with similar accretion properties will be those with high spin.

\section{Discussion}

Several aspects of the X-ray broad-band spectrum of PG1247 are in agreement with the possibility that this high-redshift QSO is 
accreting close to or above the Eddington limit, independent of the model adopted to reproduce the overall spectral shape:

- The photon index is very soft: $\Gamma \sim 2.3-2.4$ for all models. These values are consistent with those expected for a nearly Eddington accreting SMBH, given the relation observed in individual, variable, local AGN (e.g., Perola et al. 1986; Vaughan \& Edelson 2001; Vignali et al. 2008; Puccetti et al. 2014), and in samples of low- and high-redshift AGN (Shemmer et al. 2008; Risaliti et al. 2009; Brightman et al. 2013). The caveat is that the parameter space above the Eddington limit is currently unexplored. Unfortunately, owing to the limited data quality and the lack of simultaneity between the XMM-Newton and NuSTAR data, we cannot test whether PG1247 shows the softer when brighter behavior within the flux variation observed between the XMM-Newton and NUSTAR data.

- The Fe emission line is most likely produced by ionized gas in the accretion disk (the rest-frame energy of $6.4 \mathrm{keV}$ is ruled out at $90 \%$ c.l.), as observed in the stacked spectrum of large samples of AGN accreting close to the Eddington limit (Iwasawa et al. 2012). The EW of the line $\left(E W=168_{-150}^{+198} \mathrm{eV}\right)$ is consistent, within the errors, with the value expected taking into account the high reflection factor derived from PEXRAV (possibly due to variability, see below) and the X-ray Baldwin effect ( $E W \sim 20-30 \mathrm{eV}$ intrinsic, to be rescaled by a reflection parameter $R=3.8$ ).

- The residuals above the expected energies of $\mathrm{Fe} \mathrm{K} \alpha$ suggest the presence of an outflowing disk wind, as predicted by super-Eddington accretion models (e.g., Zubovas \& King 2012), and observed in a handful of high-redshift QSOs accreting close to Eddington (Chartas et al. 2003; Lanzuisi et al. 2012; Vignali et al. 2015). The presence of ionized material surrounding the SMBH is indeed one of the characteristics of highly accreting BHs (see, e.g., Ballantyne et al. 2011).

\subsection{Reflection and accretion}

The intensity of the reflection component is usually thought to be anti-correlated with the flux state, and therefore $\lambda_{\mathrm{Edd}}$, so that sources accreting closer to the Eddington limit show lower levels of reflection (see, e.g., Fabian et al. 2012; Keek \& Ballantyne 2016). Therefore, the super-Eddington nature of PG1247 and the exceptional reflection component observed in its spectrum seem in tension. However, with the exception of model 1, which requires an unphysical value of the reflection fraction $(R>1)$, each of the other models discussed in Sect. 5 offers an explanation, even though the conclusions are strongly model-dependent.

In PG1247, the SMBH is on average accreting close to or above Eddington, while the XMM-Newton observation of 2003 can be considered a drop in the X-ray flux of a factor of $\sim 2$ with respect to the average, and a factor of $\sim 3$ with respect to the ROSAT measurements. The cold reflection scenario is therefore able to explain the exceptionally high reflection component in terms of variability (model 2 ): the source was a factor of $\sim 3$ fainter at the time of the XMM-Newton observation than during the ROSAT observation. In this scenario the super-Eddington accretion rate has nothing to do with the presence of a strong reflection component, which is only a light echo of the continuum level as it was 1200 days before the XMM-Newton observation. A reflection fraction $R=3-4$ in the $X M M-N e w t o n$ data is therefore in agreement with this scenario, while the reflection fraction for the NuSTAR data in model 2 is consistent with $R=1$ within the errors.

We note that the value $R=1$ in the PEXRAV model is defined relative to the reflection produced by an infinite slab of cold material illuminated by the corona. If instead the reflector is the inner wall of the torus, we do not expect a reflection as high as $R=1$ (the total solid angle seen by the reflector must be $<2 \pi$ sr). However, if we imagine a torus with a small half opening angle and large height (e.g., the one described in Ikeda et al. 2009), $R$ could be close to $\sim 1$. Furthermore, if the reflection component is produced by cold material at the inner edge of the obscuring torus, the time delay between continuum and cold reflection variability would imply a radius of the torus inner edge of $\sim 1 \mathrm{pc}$, roughly in agreement with the expected sublimation radius $r_{\text {in }}=1-4$ pc (Barvainis 1987; Kishimoto et al. 2007) given the UV luminosity of $\lambda L_{\lambda}(1350 \AA) \sim 4 \times 10^{47} \mathrm{erg} \mathrm{s}^{-1}$ (Trevese et al. 2014) and with the torus half-light radius expected from the bolometric luminosity, i.e., $1-10 \mathrm{pc}$ at $10^{48} \mathrm{erg} \mathrm{s}^{-1}$ (Burtscher et al. 2013).

The ionized absorber scenario (model 3) is able to explain the broad-band shape observed in PG1247 without the need of any reflection component. The spectral curvature is due to the effect of a mildly ionized $\left(\log \xi \sim 2.4 \mathrm{erg} \mathrm{cm} \mathrm{s}^{-1}\right)$ dense absorber $\left(N_{\mathrm{H}} \sim 1 \times 10^{24} \mathrm{~cm}^{-2}\right)$ with a covering factor of $C F \sim 0.6$ superimposed on a simple power law with a high-energy cutoff $E_{\text {cut }} \sim 100 \mathrm{keV}$. The addition of a standard cold reflection with $R=1$ would reduce the required covering factor to $C F \sim 0.4$. In this case, the link between the shape of the X-ray spectrum of PG1247 and its extreme accretion properties would be in the presence of ionized, outflowing material close to the $\mathrm{SMBH}$, a clear prediction of super-Eddington accretion theories that needs to be investigated with higher $\mathrm{S} / \mathrm{N}$ spectra in the region of the $\mathrm{Fe} \mathrm{K} \alpha$ emission line.

Finally, the relativistic reflection scenario (model 4) is able to explain the strong reflection component with an extreme geometry of the system, i.e., a rather small inclination angle $\left(i<30^{\circ}\right)$, a small height of the corona $\left(h<3.5 r_{\mathrm{G}}\right)$, and a maximally rotating $\mathrm{BH}(a \sim 0.97)$. The face-on geometry required to explain the strong reflection may actually affect the SMBH mass measurements, in particular requiring an increase in both the SE and RM mass estimates by a factor of up to 5 for very small angles (Pancoast et al. 2014). This would reduce the Eddington ratio of PG1247 by the same amount.

\section{2. $E_{\mathrm{cut}}$ and the properties of the corona}

The confidence contours for $\Gamma$ and $E_{\text {cut }}$ measured from the different models are shown in Fig. 10. The results on $E_{\text {cut }}$ obtained from model 1 (left panel) are not considered here since, as discussed above, this model must be considered unrealistic. The best-fit $E_{\text {cut }}$ measured with model 2 (central panel) is $E_{\text {cut }} \sim$ $160 \mathrm{keV}$, but it is only loosely constrained to be $E_{\text {cut }} \lesssim 600 \mathrm{keV}$. For model 3 instead, we clearly constrain, for the first time at such high redshift, a rather low cutoff (right panel). Finally, for model 4 the $E_{\text {cut }}$ is unconstrained and therefore fixed in the spectral fit.

The high-energy cutoff measured in model 3 is consistent with the average $E_{\text {cut }}$ observed at low redshift (e.g., Malizia et al. 2014: $\left\langle E_{\text {cut }}\right\rangle=128 \mathrm{keV}$, with standard deviation $\sigma=46 \mathrm{keV}$. This rather low value would imply a low plasma temperature of the Comptonizing region $\left(k T_{\mathrm{e}}=E_{\text {cut }} / 2\right.$ for $\tau \lesssim 1$ and large $\left.\lambda_{\text {Edd }}\right)$. Indeed, using Eq. (1) of Petrucci et al. (2001) and the 

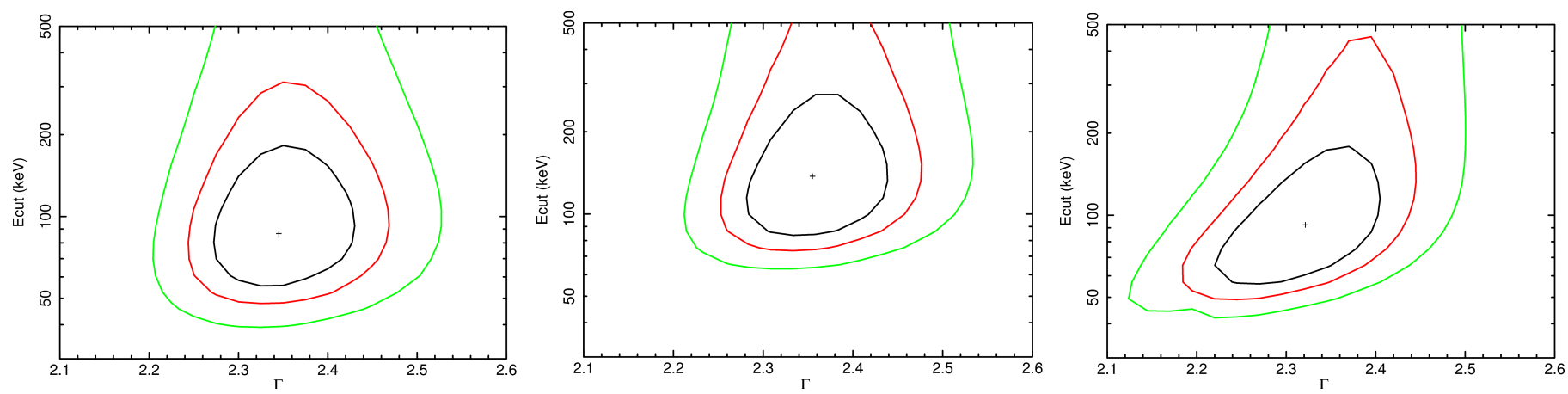

Fig. 10. Confidence contours (at 68,90 , and $99 \%$ c.1.) of $\Gamma$ vs. $E_{\text {cut }}$ (rest frame) parameters for model 1 (left), model 2 (center), and model 3 (right).

derived $E_{\text {cut }}$ and $\Gamma$ values, we can derive the optical depth of the corona. Using the results from model 3, the optical depth is $\tau \sim 1.3$, i.e., the corona is optically thick. We stress, however, that - as shown in Petrucci et al. (2001) - the $k T_{\mathrm{e}}$ values derived from PEXRAV are in general underestimated, while the $\tau$ is overestimated with respect to a realistic, anisotropic Comptonization model, and therefore these results must be taken with caution.

We stress that the result obtained for model 3 is, to our best knowledge, the first detection of a high-energy cutoff at such high redshift. The rather low temperature derived from the highenergy cutoff and the high compactness of the corona $(\ell \gtrsim 10$, with $\ell$ defined as $\ell=L \sigma_{\mathrm{T}} / R m_{\mathrm{e}} c^{3}$, Guilbert et al. 1983) are in agreement with our current understanding of the heating and thermalization mechanisms operating in the corona where large powers are dissipated in the physically compact regions surrounding the SMBH (Fabian et al. 2015).

PG1247 is, therefore, a clear example of how NUSTAR is opening up the possibility of measuring the fundamental parameters of coronal emission $\left(\ell, \tau\right.$, and $\left.k T_{\mathrm{e}}\right)$ beyond the local Universe, thanks to sensitive hard X-ray observations. However, a campaign of deep XMM-Newton observations, coupled with simultaneous hard X-ray data from NUSTAR, are required in order to understand which of the models discussed in Sect. 5 best represents the observed X-ray spectrum of PG1247, e.g., distinguishing between cold and relativistic reflection or clearly detecting absorption troughs associated with ionized obscuring gas.

Acknowledgements. We thank the anonymous referee for constructive comments that have helped us to improve the quality of the paper. G.L. thanks F. Gastaldello for useful insights on NUSTAR background issues, E. Dalessandro for advice about HST data, and O. Shemmer for help with the Swift data. G.L. acknowledges financial support from the CIG grant "eEASY" No. 321913 and from ASI-INAF 2014-045-R.0 and ASI/INAF I/037/12/0-011/13 grants. A.C.F. acknowledges support from ERC grant 340442. FEB acknowledges support from CONICYT-Chile (Basal-CATA PFB-06/2007, FONDECYT Regular 1141218, "EMBIGGEN" Anillo ACT1101), the Ministry of Economy, Development, and Tourism's Millennium Science Initiative through grant IC120009, awarded to The Millennium Institute of Astrophysics, MAS. W.N.B. and B.L. acknowledges support from Caltech NuSTAR subcontract 44A-1092750. This work made use of data from the NUSTAR mission, a project led by the California Institute of Technology, managed by the Jet Propulsion Laboratory, and funded by NASA. This research also made use of the NUSTAR Data Analysis Software (NuSTARDAS) jointly developed by the ASI Science Data Center (ASDC, Italy) and the California Institute of Technology (USA).

\section{References}

Abazajian, K. N., Adelman-McCarthy, J. K., Agüeros, M. A., et al. 2009, ApJS, 182,543

Ai, Y. L., Yuan, W., Zhou, H. Y., Wang, T. G., \& Zhang, S. H. 2011, ApJ, 727, 31

Ballantyne, D. R., McDuffie, J. R., \& Rusin, J. S. 2011, ApJ, 734, 112

Barvainis, R. 1987, ApJ, 320, 537
Bianchi, S., Guainazzi, M., Matt, G., \& Fonseca Bonilla, N. 2007, A\&A, 467, L19

Boller, T., Freyberg, M. J., Trümper, J., et al. 2016, A\&A, 588, A103

Brightman, M., Silverman, J. D., Mainieri, V., et al. 2013, MNRAS, 433, 2485

Burtscher, L., Meisenheimer, K., Tristram, K. R. W., et al. 2013, A\&A, 558, A149

Cackett, E. M., Zoghbi, A., Reynolds, C., et al. 2014, MNRAS, 438, 2980

Chartas, G., Brandt, W. N., \& Gallagher, S. C. 2003, ApJ, 595, 85

Chartas, G., Kochanek, C. S., Dai, X., Poindexter, S., \& Garmire, G. 2009, ApJ, 693, 174

Comastri, A., Fiore, F., Guainazzi, M., et al. 1998, A\&A, 333, 31

Dadina M., Vignali, C., Cappi, M., et al. 2016, A\&A, submitted

Dauser, T., Garcia, J., Wilms, J., et al. 2013, MNRAS, 430, 1694

Dauser, T., García, J., Parker, M. L., Fabian, A. C., \& Wilms, J. 2014, MNRAS, 444, L100

Dauser, T., Garcia, J., Walton, D., et al. 2016, A\&A, 590, A76

Eddington, A. S. 1916, MNRAS, 77, 16

Fabian, A. C., Zoghbi, A., Wilkins, D., et al. 2012, MNRAS, 419, 116

Fabian, A. C., Lohfink, A., Kara, E., et al. 2015, MNRAS, 451, 4375

García, J., \& Kallman, T. R. 2010, ApJ, 718, 695

García, J., Dauser, T., Lohfink, A., et al. 2014, ApJ, 782, 76

George, I. M., \& Fabian, A. C. 1991, MNRAS, 249, 352

Gibson, R. R., Jiang, L., Brandt, W. N., et al. 2009, ApJ, 692, 758

Guilbert, P. W., Fabian, A. C., \& Rees, M. J. 1983, MNRAS, 205, 593

Harrison, F. A., Craig, W. W., Christensen, F. E., et al. 2013, ApJ, 770, 103

Iwasawa, K., \& Taniguchi, Y. 1993, ApJ, 413, L15

Iwasawa, K., Mainieri, V., Brusa, M., et al. 2012, A\&A, 537, A86

Kalberla, P. M. W., Burton, W. B., Hartmann, D., et al. 2005, A\&A, 440, 775

Kallman, T., \& Bautista, M. 2001, ApJS, 133, 221

Keek, L., \& Ballantyne, D. R. 2016, MNRAS, 456, 2722

King, A. 2003, ApJ, 596, L27

Kishimoto, M., Hönig, S. F., Beckert, T., \& Weigelt, G. 2007, A\&A, 476, 713

Kormendy, J., \& Ho, L. C. 2013, ARA\&A, 51, 511

Lanzuisi, G., Giustini, M., Cappi, M., et al. 2012, A\&A, 544, A2

Lanzuisi, G., Ponti, G., Salvato, M., et al. 2014, ApJ, 781, 105

Laurent, P., \& Titarchuk, L. 2011, ApJ, 727, 34

Luo, B., Brandt, W. N., Hall, P. B., et al. 2015, ApJ, 805, 122

Lusso, E., Comastri, A., Simmons, B. D., et al. 2012, MNRAS, 425, 623

Madsen, K. K., Harrison, F. A., Markwardt, C. B., et al. 2015, ApJS, 220, 8

Magdziarz, P., \& Zdziarski, A. A. 1995, MNRAS, 273, 837

Markowitz, A., Reeves, J. N., \& Braito, V. 2006, ApJ, 646, 783

Matt, G., Perola, G. C., \& Piro, L. 1991, A\&A, 247, 25

Nandra, K., George, I. M., Turner, T. J., \& Fukazawa, Y. 1996, ApJ, 464, 165

Nandra, K., George, I. M., Mushotzky, R. F., Turner, T. J., \& Yaqoob, T. 1997, ApJ, 476, 70

Nardini, E., Reeves, J. N., Gofford, J., et al. 2015, Science, 347, 860

Nobuta, K., Akiyama, M., Ueda, Y., et al. 2012, ApJ, 761, 143

Page, K. L., Reeves, J. N., O’Brien, P. T., Turner, M. J. L., \& Worrall, D. M. 2004, MNRAS, 353, 133

Pancoast, A., Brewer, B. J., Treu, T., et al. 2014, MNRAS, 445, 3073

Perola, G. C., Piro, L., Altamore, A., et al. 1986, ApJ, 306, 508

Peterson, B. M., Wanders, I., Bertram, R., et al. 1998, ApJ, 501, 82

Petrucci, P. O., Haardt, F., Maraschi, L., et al. 2001, ApJ, 556, 716

Pounds, K. A., Reeves, J. N., King, A. R., et al. 2003a, MNRAS, 345, 705

Pounds, K. A., King, A. R., Page, K. L., \& O'Brien, P. T. 2003b, MNRAS, 346, 1025

Protassov, R., van Dyk, D. A., Connors, A., Kashyap, V. L., \& Siemiginowska, A. 2002, ApJ, 571, 545

Puccetti, S., Comastri, A., Fiore, F., et al. 2014, ApJ, 793, 26 
Reeves, J. N., O’Brien, P. T., \& Ward, M. J. 2003, ApJ, 593, L65

Reeves, J., Done, C., Pounds, K., et al. 2008, MNRAS, 385, L108

Reis, R. C., \& Miller, J. M. 2013, ApJ, 769, L7

Reis, R. C., Reynolds, M. T., Miller, J. M., \& Walton, D. J. 2014, Nature, 507, 207

Reynolds, M. T., Walton, D. J., Miller, J. M., \& Reis, R. C. 2014, ApJ, 792, L19

Ricci, C., Paltani, S., Awaki, H., et al. 2013, A\&A, 553, A29

Risaliti, G., Young, M., \& Elvis, M. 2009, ApJ, 700, L6

Saez, C., \& Chartas, G. 2011, ApJ, 737, 91

Saturni, F. G., Trevese, D., Vagnetti, F., Perna, M., \& Dadina, M. 2016, A\&A, 587, A43

Schulze, A., \& Wisotzki, L. 2010, A\&A, 516, A87

Shemmer, O., Brandt, W. N., Netzer, H., Maiolino, R., \& Kaspi, S. 2006, ApJ, 646, L29

Shemmer, O., Brandt, W. N., Netzer, H., Maiolino, R., \& Kaspi, S. 2008, ApJ, 682,81

Shemmer, O., Brandt, W. N., Paolillo, M., et al. 2014, ApJ, 783, 116

Shen, Y., Richards, G. T., Strauss, M. A., et al. 2011, ApJS, 194, 45

Shih, D. C., Iwasawa, K., \& Fabian, A. C. 2002, MNRAS, 333, 687

Shu, X. W., Wang, J. X., Yaqoob, T., Jiang, P., \& Zhou, Y. Y. 2012, ApJ, 744, L21

Steffen, A. T., Strateva, I., Brandt, W. N., et al. 2006, AJ, 131, 2826

Suh, H., Hasinger, G., Steinhardt, C., Silverman, J. D., \& Schramm, M. 2015, ApJ, 815, 129
Tombesi, F., Cappi, M., Reeves, J. N., et al. 2010, A\&A, 521, A57

Tombesi, F., Cappi, M., Reeves, J. N., et al. 2013, MNRAS, 430, 1102

Trevese, D., Paris, D., Stirpe, G. M., Vagnetti, F., \& Zitelli, V. 2007, A\&A, 470, 491

Trevese, D., Perna, M., Vagnetti, F., Saturni, F. G., \& Dadina, M. 2014, ApJ, 795, 164

Uttley, P., Cackett, E. M., Fabian, A. C., Kara, E., \& Wilkins, D. R. 2014, A\&ARv, 22, 72

Vasudevan, R. V., Fabian, A. C., Reynolds, C. S., et al. 2016, MNRAS, 458, 2012 Vaughan, S., \& Edelson, R. 2001, ApJ, 548, 694

Vestergaard, M. 2003, ApJ, 599, 116

Vignali, C., Piconcelli, E., Bianchi, S., \& Miniutti, G. 2008, MNRAS, 388, 761

Vignali, C., Iwasawa, K., Comastri, A., et al. 2015, A\&A, 583, A141

Volonteri, M. 2012, Science, 337, 544

Walton, D. J., Nardini, E., Fabian, A. C., Gallo, L. C., \& Reis, R. C. 2013 , MNRAS, 428, 2901

Weymann, R. J., Williams, R. E., Peterson, B. M., \& Turnshek, D. A. 1979, ApJ, 234,33

Wik, D. R., Hornstrup, A., Molendi, S., et al. 2014, ApJ, 792, 48

Young, M., Brandt, W. N., Xue, Y. Q., et al. 2012, ApJ, 748, 124

Zoghbi, A., Fabian, A. C., Reynolds, C. S., \& Cackett, E. M. 2012, MNRAS, 422, 129

Zubovas, K., \& King, A. 2012, ApJ, 745, L34

Zubovas, K., \& King, A. 2013, ApJ, 769, 51 